\section{Trauma psicológico en la atención primaria: orientaciones de manejo}

\author{
RODRIGO A. FIGUEROA ${ }^{1,2}$, PAULA F. CORTÉS ${ }^{2}$, \\ LUIGI ACCATINO ${ }^{1}$, RICHARD SORENSEN ${ }^{3}$
}

\section{Management of psychological trauma in primary care}

Exposure to traumatic events is frequent in the general population and psychiatric sequelae such as post-traumatic stress disorders are common. The symptoms of psychiatric sequelae after trauma are vague, with multiple psychological and physical symptoms, which can confuse the health care professional. This paper seeks to facilitate the work in primary care, providing practical information about the diagnosis, initial management and referral of patients who have suffered traumatic experiences. Some early interventions and treatments are suggested.

(Rev Med Chile 2016; 144: 643-655)

Key words: Practice Guidelines as Topic; Primary Health Care; Psychological trauma; Stress Disorders, Post-Traumatic; Trauma and Stressor Related Disorders.
'Departamento de Psiquiatría, Escuela de Medicina, Pontificia Universidad Católica de Chile.

${ }^{2}$ Centro de Investigación para la Gestión Integrada de Desastres Naturales (CIGIDEN), Santiago, Chile. ${ }^{3}$ United States Air Force.

Fuente de apoyo financiero: Este artículo recibió apoyo financiero de la Corporación Nacional de Investigación, Ciencia y Tecnología (CONICYT) a través del Centro de Investigación para la Gestión Integrada de Desastres Naturales (CIGIDEN)-FONDAP No 15110017/2011. La institución no tuvo influencia en el diseño, preparación o revisión del manuscrito. No se declaran conflictos de interés por parte de ninguno de los autores.

Recibido el 24 de julio de 2015, aceptado 1 de abril de 2016 .

Correspondencia a:

Dr. Rodrigo A. Figueroa

Centro de Salud Mental, Centro

Médico San Joaquín, Red de Salud UC

Christus.

Av. Vicuña Mackenna 4686, Macul,

Santiago, Chile.

rfiguerc@uc.cl

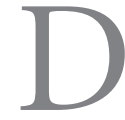

e acuerdo al DSM-5 $5^{1}$, trauma es definido como cualquier situación en la que una persona se vea expuesta a escenas de muerte real o inminente, lesiones físicas graves o agresión sexual, ya sea en calidad de víctima directa, cercano a la víctima o testigo. El CIE-11, próximo a ser publicado, lo conceptualiza como cualquier exposición a una situación estresante de naturaleza excepcionalmente amenazante $u$ horrorizante que probablemente producirá un malestar profundo en la mayoría de las personas ${ }^{2,3}$.

La frecuencia de las experiencias traumáticas en la población general es alta. En Chile aproximadamente $40 \%$ de la población ha experimentado un trauma alguna vez en su vida ${ }^{4}$, lo que ubica a nuestro país en una situación intermedia comparado con México (80\%), Estados Unidos de Norteamérica (50\%) y Alemania $(20 \%)^{5-8}$.

Los traumas pueden dejar varias secuelas psiquiátricas como Trastorno de Estrés Postraumático (TEPT), depresión y trastornos de ansiedad, entre $\operatorname{otros}^{9}$ (Tabla 1). La alta comorbilidad del TEPT $^{10}$, su multiplicidad de formas de presentación ${ }^{11,12}$ y la resistencia natural de los pacientes y clínicos a hablar del trauma tornan común el subdiagnóstico de éste, a pesar de que se han descrito prevalencias de alrededor de $12 \%$ en la Atención Primaria de Salud (APS) ${ }^{13-15}$.

Del total de personas que vive un trauma, aproximadamente $14 \%$ desarrollará un $\mathrm{TEPT}^{10}$, siendo mayor en aquellos ocasionados por la acción intencional de terceros (ej. violaciones, asaltos, guerra, terrorismo, etc.). En Chile la mayoría de los casos de TEPT son producto de accidentes de tránsito o muerte violenta de un ser querido ${ }^{4}$, ocasionando una pérdida de 19.869 años de vida saludable por muerte prematura o discapacidad $(\mathrm{AVISA})^{16}$, lo que ubica a este trastorno en la posición 44 del ranking de enfermedades que más aportan a la carga de enfermedad del país. En el mundo, el TEPT aporta $0,2 \%$ de la carga global de enfermedad, al igual que el cáncer cervicouterino ${ }^{17}$. Además de los costos individuales, las secuelas emocionales de los traumas producen múltiples dificultades psicosociales como problemas maritales ${ }^{18,19}$, legales ${ }^{10}$, disfunción familiar ${ }^{20,21}$, desempleo, abuso de alcohol $^{8}$ y suicidio ${ }^{22}$. Su prevalencia varía mucho según el país estudiado: 
Tabla 1. Trastornos del espectro postraumático, basado en Moreau y Zisook (2002) ${ }^{9}$

Trastornos de adaptación
Trastorno de estrés agudo
Somatización (trastornos somatomorfos)
Duelo complicado
Trastornos de la personalidad
Trastornos del ánimo
Trastornos de ansiedad
Trastorno de estrés postraumático
$\quad$ Subclínico
Complicado
Trastornos disociativos*
*Aunque no se menciona en el artículo de Moreau y Zisook
(2002) ${ }^{9}$, los trastornos disociativos se consideran una secuela
de eventos traumáticos extremos ${ }^{65}$.

mientras que en México 12,2\% de la población ha presentado TEPT alguna vez en su vida ${ }^{5}$, en Chile sólo lo ha hecho $4,4 \%{ }^{4}$.

Entre los factores de riesgo conocidos se encuentra el sexo femenino, los antecedentes psiquiátricos ${ }^{23}$, una alta percepción de riesgo vital durante el trauma y posteriormente al trauma una baja percepción de apoyo social, presencia de secuelas físicas y alta percepción de estrés psicosocial $^{24}$. Adicionalmente, se han encontrado factores de riesgo relacionados con el bajo nivel socioeconómico, educacional, de salud y de seguridad social. Es importante señalar, sin embargo, que ninguno de estos factores incrementa el riesgo tanto como para predecir quién desarrollará un TEPT $^{24,25}$. Aproximadamente $40 \%$ de las personas que desarrolla un Trastorno de Estrés Agudo durante el primer mes post-trauma evoluciona hacia un TEPT ${ }^{26-28}$. Un tercio de aquellos que desarrollan un TEPT continúa sintomático una década después ${ }^{28}$.

Dada la alta frecuencia de los traumas en la población general y su significativo impacto en salud pública es importante que los profesionales de la APS estén sensibilizados respecto al tema y sean capaces de sospechar, detectar y manejar las reacciones inmediatas y secuelas de largo plazo del trauma. Los profesionales APS se encuentran en una posición crítica del sistema de salud que los transforma en un eslabón esencial en el continuo de cuidados que requiere esta población. Esto cobra aún mayor relevancia en un país como Chile, frecuentemente afectado por desastres naturales como terremotos e inundaciones, donde la respuesta comunitaria es fundamental ${ }^{29,30}$. En esa línea, este trabajo tiene como objetivo entregar información práctica para el personal APS, de modo de facilitar su trabajo cuando les corresponda atender a personas que hayan vivido experiencias traumáticas (Figueroa RA, Bas C, Padilla O, Cortés PF. Social determinants of health and PTSD after the 2010 earthquake in Chile: Datos no Observaciones no publicadas. XVI Congreso Mundial de Psiquiatría, Madrid, 2014).

\section{Reacciones emocionales y trayectorias después de un trauma}

Durante los primeros días o semanas después de una situación traumática la mayoría de las personas experimenta reacciones emocionales intensas y perturbadoras, como insomnio, labilidad emocional, irritabilidad, recuerdos intrusivos sobre el evento y/o aversión a claves que recuerden el trauma, entre otras ${ }^{31}$ (Tabla 2). Todas estas reacciones se consideran "reacciones normales frente a una situación anormal" y no constituyen necesariamente un signo de algún trastorno. Se han descrito cuatro trayectorias de evolución ${ }^{32}:$ a) resiliente, observada en personas que son capaces de continuar sus rutinas familiares, laborales y sociales con un mínimo nivel de perturbación (35-65\%); b) de recuperación, en personas que luego de un período de fuerte perturbación inicial se recuperan al cabo de algunos meses (15-25\%); c) retardada, cuando luego de varios meses de mantener un funcionamiento relativamente normal la persona enferma (0-15\%); y d) cróni$\mathrm{ca}$, cuando rápidamente se produce un deterioro del funcionamiento y el paciente no se vuelve a recuperar (5-30\%) (Figura 1). Adicionalmente y en el largo plazo, un grupo significativo de los afectados por experiencias traumáticas podría desarrollar "crecimiento postraumático", fenómeno caracterizado por cambios positivos en la relación con uno mismo, los demás y la filosofía de vida ${ }^{33}$. Lamentablemente, hoy no es posible predecir durante los primeros días y semanas después de un trauma quiénes desarrollarán una u otra trayectoria, ni si desarrollarán crecimiento 
Tabla 2. Reacciones emocionales, cognitivas, físicas e interpersonales comunes frente a experiencias traumáticas recientes

\section{Reacciones emocionales}

- Estado de shock

- Terror

- Irritabilidad

- Sentimiento de culpa (hacia sí mismo y hacia otros)

- Rabia

- Dolor o tristeza

- Embotamiento emocional

- Sentimientos de impotencia

- Pérdida del disfrute derivado de actividades familiares

- Dificultad para sentir alegría

- Dificultad para experimentar sentimientos de amor y cariño por otros

\section{Reacciones cognitivas}

- Dificultades para concentrarse

- Dificultades para tomar decisiones

- Dificultades de memoria

- Incredulidad

- Confusión

- Pesadillas

- Autoestima decaída

- Noción de autoeficacia disminuida

- Pensamientos culposos

- Pensamientos o recuerdos intrusivos sobre el trauma

- Preocupación

- Disociación (ej. visión túnel, estado crepuscular de conciencia, sensación de irrealidad, dificultades para recordar, sensación de separación del cuerpo, etc.)

\section{Reacciones físicas}

- Fatiga, cansancio

- Insomnio

- Taquicardia o palpitaciones

- Sobresaltos

- Exaltación

- Incremento de dolores físicos

- Reducción de la respuesta inmune

- Dolores de cabeza

- Perturbaciones gastrointestinales

- Reducción del apetito

- Reducción del deseo sexual

- Vulnerabilidad a la enfermedad

\section{Reacciones interpersonales}

- Incremento en conflictos interpersonales

- Retraimiento social

- Reducción en las relaciones íntimas

- Alienación

- Dificultades en el desempeño laboral o escolar

- Reducción de la satisfacción

- Desconfianza

- Externalización de la culpa

- Externalización de la vulnerabilidad

- Sensación de abandono o rechazo

- Sobreprotección

Adaptado de Common Reactions After Trauma, del National Center for PTSD, U.S Department of Veterans Affairs64.

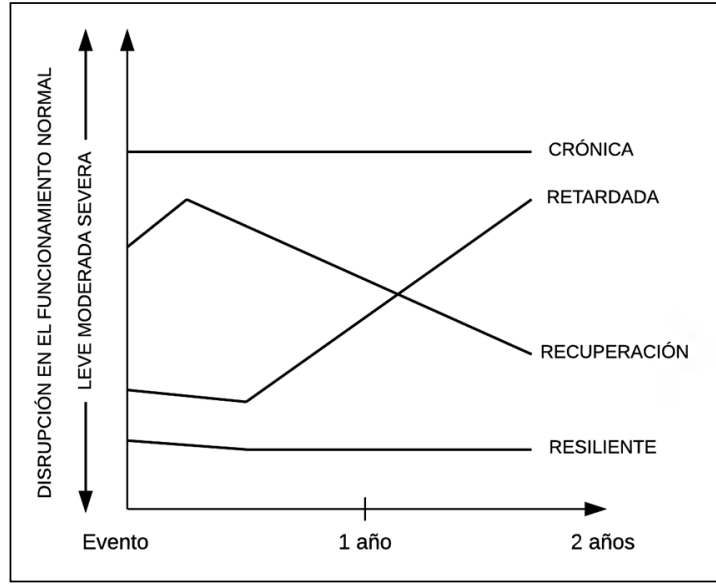

Figura 1. Trayectorias típicas de disrupción en el funcionamiento normal a lo largo del tiempo luego de un evento potencialmente traumático (adaptado con permiso de Bonano, 2004).

postraumático, por lo que el manejo del trauma será inespecífico en las primeras dos semanas y recién incorporará técnicas específicas a partir de la tercera ${ }^{34}$.

\section{Manejo durante las primeras dos semanas post-trauma}

Durante las primeras dos semanas luego de un evento traumático el tratamiento de elección son los Primeros Auxilios Psicológicos (PAP), que corresponden a "una respuesta humana de apoyo a otro ser humano que está sufriendo y que puede necesitar ayuda" 35,36 . Se trata de una estrategia de ayuda humanitaria inespecífica que se considera de elección por su bajo riesgo de iatrogenia, por su simpleza (no necesita ser profesional de la salud mental quien la aplique) y porque promueve varios factores "informados en la evidencia" que de acuerdo a varios expertos favorecerían la resiliencia (seguridad, calma, autoeficacia, conexión y esperanza $)^{37}$. Es una técnica poco estructurada, adaptada al paciente y su cultura, pero que en la práctica, y con fines operativos, la resumiremos en cinco acciones: a) ofrecer un tiempo razonable de escucha activa (10-20 min), que puede ser definida como "poner toda la atención de uno en la otra persona y comunicarle que estamos interesados y preocupados" a través de un lenguaje corporal atento, un seguimiento adecuado de lo 
que el paciente dice y conductas reflejo ${ }^{38}$, como copiar o repetir las posturas, gestos o palabras que la persona utiliza; b) ofrecer ayuda concreta para lograr la calma, lo que puede efectuarse con técnicas de reentrenamiento de la respiración ${ }^{39}$ apoyadas incluso por aplicaciones móviles ${ }^{40,41}$, contracturas musculares progresivas de Jacob$\operatorname{son}^{42,43} \mathrm{u}$ otras técnicas de relajación (Tabla 3); c) ofrecer apoyo en sistematización y priorización de necesidades inmediatas como contacto con familiares, información, protección financiera, asistencia legal, abrigo, etc.; d) ofrecer apoyo para satisfacer las necesidades prioritarias, activando las redes de apoyo y de seguridad social disponibles; y e) ofrecer psicoeducación respecto a las reacciones normales frente al trauma, cómo ayudarse y ayudar a otros, cuáles son los signos de alarma y dónde buscar ayuda. Como podrá notar el lector, cada una de estas acciones es una oferta, que sólo será implementada si el paciente la acepta voluntariamente. En la Tabla 4 se pueden ver ejemplos típicos de frases apropiadas para cada una de las cinco acciones de PAP mencionadas.

Aunque los PAP son efectivos para lograr restituir la calma en la mayoría de las personas, en algunos casos no serán suficientes. En esos casos excepcionales podría justificarse el uso de psicofármacos como antipsicóticos o benzodiazepinas en dosis bajas por períodos de pocas semanas. Es muy importante tener cuidado con el uso de benzodiazepinas porque podrían aumentar la incidencia de TEPT, desinhibir la agresividad/ impulsividad y facilitar o agravar un cuadro de abuso o dependencia de sustancias, además de interferir el proceso de psicoterapia cuando se requiera tratamiento definitivo. Su uso prolongado también aumenta el riesgo de caídas, deterioro de la memoria, accidentes, problemas de aprendizaje y sueño no reparador, además de ser inefectivas en el tratamiento de las secuelas emocionales crónicas del trauma ${ }^{44}$. Adicionalmente, existe alguna evidencia no concluyente señalando que podrían aumentar el riesgo de demencias ${ }^{45}$, por lo que desaconsejamos enfáticamente utilizar esta familia de medicamentos como primera opción para el manejo agudo del trauma reciente. La quetiapina en dosis de $25 \mathrm{mg}$ al día dividida en tres dosis u otros antipsicóticos a titular según respuesta son una mejor alternativa, aunque también deben ser utilizados por períodos breves. Sugerimos derivar inmediatamente a urgencia psiquiátrica a aquellos pacientes en que exista ideación suicida o agresividad marcada hacia terceros, catatonía, psicosis o algún trastorno psiquiátrico previo grave que pueda descompensarse en forma severa, ya que todas esas condiciones requieren manejo especializado (Figura 2).

Por último, es recomendable aplicar durante las primeras dos semanas post-trauma alguna escala autoaplicada de síntomas de TEPT y depresión como la PTSD Checklist $(\mathrm{PCL})^{46}$ y el Inventario de Depresión de Beck-II (BDI-II) ${ }^{47}$.

\section{Tabla 3. Técnica de reentrenamiento de la respiración, adaptada de Foa, Hembree y Rothbaum (2007) ${ }^{40}$}

\footnotetext{
Explíquele al paciente detalladamente la lógica del ejercicio:

"La forma como respiramos modifica nuestras emociones. Cuando botamos el aire nos relajamos más que cuando inspiramos (al contrario de lo que se suele creer), por lo que podemos entrar en un estado de calma si prolongamos el tiempo en que nuestros pulmones están vacíos.

Explíquele y demuéstrele al paciente la mecánica del ejercicio:

"El ejercicio se trata de que usted inspire en cuatro tiempos, exhale en cuatro tiempos y aguante cuatro tiempos más antes de volver a inspirar". Ahora mire como yo lo hago... [hágalo usted mismo]

Acompañe al paciente a hacer el ejercicio:

"Ahora lo hará usted, y yo lo acompañaré recordándole cómo debe hacerlo. Inspire... dos, tres, cuatro... exhale, dos, tres cuatro... aguante, dos tres, cuatro..." [repita el ciclo por uno o dos minutos acompañando al paciente]. "Mientras exhala puede pensar en la palabra calma"

Indíquele al paciente que lo haga diariamente por diez minutos, tres veces al día (mañana, tarde y noche) y cada vez que se sienta angustiado. Puede ayudarse con la aplicación Breath pacer o Paced Breathing disponible para celulares:

"Le voy a pedir que realice este ejercicio durante 10 minutos todos los días en la mañana al despertar, después de almuerzo, antes de irse a dormir y cada vez que sienta que está comenzando a angustiarse mucho. Mientras más use esta técnica, más fácil será la próxima vez que la use"
} 


\section{Tabla 4. Ejemplos de frases útiles durante la implementación de los diferentes componentes de los Primeros Auxilios Psicológicos}

\begin{tabular}{|c|c|}
\hline Escucha activa & $\begin{array}{l}\text { "Depende de si usted quiere hacerlo o no, por supuesto... usted siempre podrá decidir, pero } \\
\text { si quiere hablar con alguien sobre esto yo encantado de escucharle" }\end{array}$ \\
\hline \multirow{3}{*}{$\begin{array}{l}\text { Promoción de calma y } \\
\text { relajación }\end{array}$} & "Ya pasó... ahora está a salvo... está en el consultorio y aquí lo vamos a cuidar" \\
\hline & "Me informó carabineros que el peligro ya pasó" \\
\hline & $\begin{array}{l}\text { "Es difícil pensar con claridad cuando uno está bajo tanto estrés... le propongo que hagamos } \\
\text { primero una pausa con unos ejercicios de relajación, y luego le ayudaré a armar un plan" }\end{array}$ \\
\hline \multirow{4}{*}{$\begin{array}{l}\text { Apoyo en la sistema- } \\
\text { tización y priorización } \\
\text { de acciones y necesi- } \\
\text { dades }\end{array}$} & "¿Cuál cree que es el problema más importante de resolver primero?" \\
\hline & "¿Qué cosas le han ayudado en el pasado cuando ha tenido que lidiar con tanto estrés?" \\
\hline & $\begin{array}{l}\text { "Obviamente son muchos problemas juntos que sería bueno ordenar para ir uno por uno... si } \\
\text { quiere puedo ayudarle a hacerlo" }\end{array}$ \\
\hline & $\begin{array}{l}\text { "Entiendo que se sienta sobrepasado. Veamos si podemos identificar al menos tres cosas sobre } \\
\text { las que tiene actualmente control para focalizarnos en ellas" }\end{array}$ \\
\hline \multirow{5}{*}{$\begin{array}{l}\text { Apoyo en el contacto } \\
\text { con familiares, amigos } \\
\text { y servicios de apoyo } \\
\text { social }\end{array}$} & $\begin{array}{l}\text { "Se ha visto que ayuda mucho para estar mejor compartir con amigos y familiares... pasar } \\
\text { tiempo juntos, acompañados" }\end{array}$ \\
\hline & $\begin{array}{l}\text { "¿Existirá algún momento en la semana en el que pueda dedicarse regularmente a compartir } \\
\text { con su familia y amigos?" }\end{array}$ \\
\hline & $\begin{array}{l}\text { "Entiendo que quizás usted desconfie de los servicios públicos de apoyo, pero si más adelante } \\
\text { cambia de opinión, quisiera que sepa cómo contactarlos" }\end{array}$ \\
\hline & $\begin{array}{l}\text { "No tengo información sobre la situación de sus hijos, pero veamos si podemos averiguar algo } \\
\text { sobre ellos en las páginas de búsqueda de personas o con la policía..." }\end{array}$ \\
\hline & $\begin{array}{l}\text { "Si le surge alguna duda más adelante, puede venir a preguntarme, o quizás se anime a llamar } \\
\text { al teléfono gratuito Salud Responde (600 } 3607777) \text {, donde alguien podrá orientarle las } 24 \mathrm{~h} \\
\text { del día, los siete días de la semana" }\end{array}$ \\
\hline \multirow[t]{8}{*}{ Psicoeducación } & $\begin{array}{l}\text { "Todas esas sensaciones que está experimentando son muy comunes después de una experien- } \\
\text { cia así. La mayoría de las personas que han vivido una situación tan estresante las presentan por } \\
\text { días e incluso semanas, pero luego se van yendo. No significan que esté perdiendo la cabeza } \\
\text { o que sea débil... ya va a ver que se le van a ir pasando" }\end{array}$ \\
\hline & $\begin{array}{l}\text { "Preocuparse de usted, y pasar un tiempo de relajo junto a su familia puede ser muy útil du- } \\
\text { rante estos días difíciles" }\end{array}$ \\
\hline & $\begin{array}{l}\text { "Afortunadamente los seres humanos solemos salir adelante de este tipo de situaciones, por } \\
\text { más duras que parezcan, por lo que tengo confianza en que usted podrá superar todo esto..." }\end{array}$ \\
\hline & $\begin{array}{l}\text { "Iremos controlando su evolución en las próximas semanas. Normalmente este malestar va } \\
\text { disminuyendo con los días y semanas, por lo que tengo confianza en que todo esto irá pasando } \\
\text { poco a poco" }\end{array}$ \\
\hline & $\begin{array}{l}\text { "Lo felicito... todo eso que ha hecho es estupendo para salir adelante. Me alegro de ver como } \\
\text { va tomando decisiones y saliendo adelante" }\end{array}$ \\
\hline & $\begin{array}{l}\text { "Durante estas dos primeras semanas evite pasar pegado a la tele viendo noticias sobre el } \\
\text { terremoto... y evite que sus niños hagan lo mismo" }\end{array}$ \\
\hline & $\begin{array}{l}\text { "Estar más emotivo, quizás con un poco de insomnio, no poder parar de pensar en lo que pasó, } \\
\text { más temeroso, más irritable, por momentos más retraído... todo eso es normal. Preocúpese si } \\
\text { con los días eso no va pasando o si aparecen ganas de morir, hacerle daño a otros, si aparecen } \\
\text { crisis de pánico repetidas, si ve que algún conocido suyo pierde contacto con la realidad... en } \\
\text { esos casos venga o tráigalo inmediatamente" }\end{array}$ \\
\hline & $\begin{array}{l}\text { "Durante estos días pueden darle más ganas de beber alcohol, fumar o quizás usar otra dro- } \\
\text { ga... Evítelo y reemplácelo por deporte, algún hobbie, yoga o meditación, que se ha visto son } \\
\text { más efectivos para superar momentos difíciles" }\end{array}$ \\
\hline
\end{tabular}




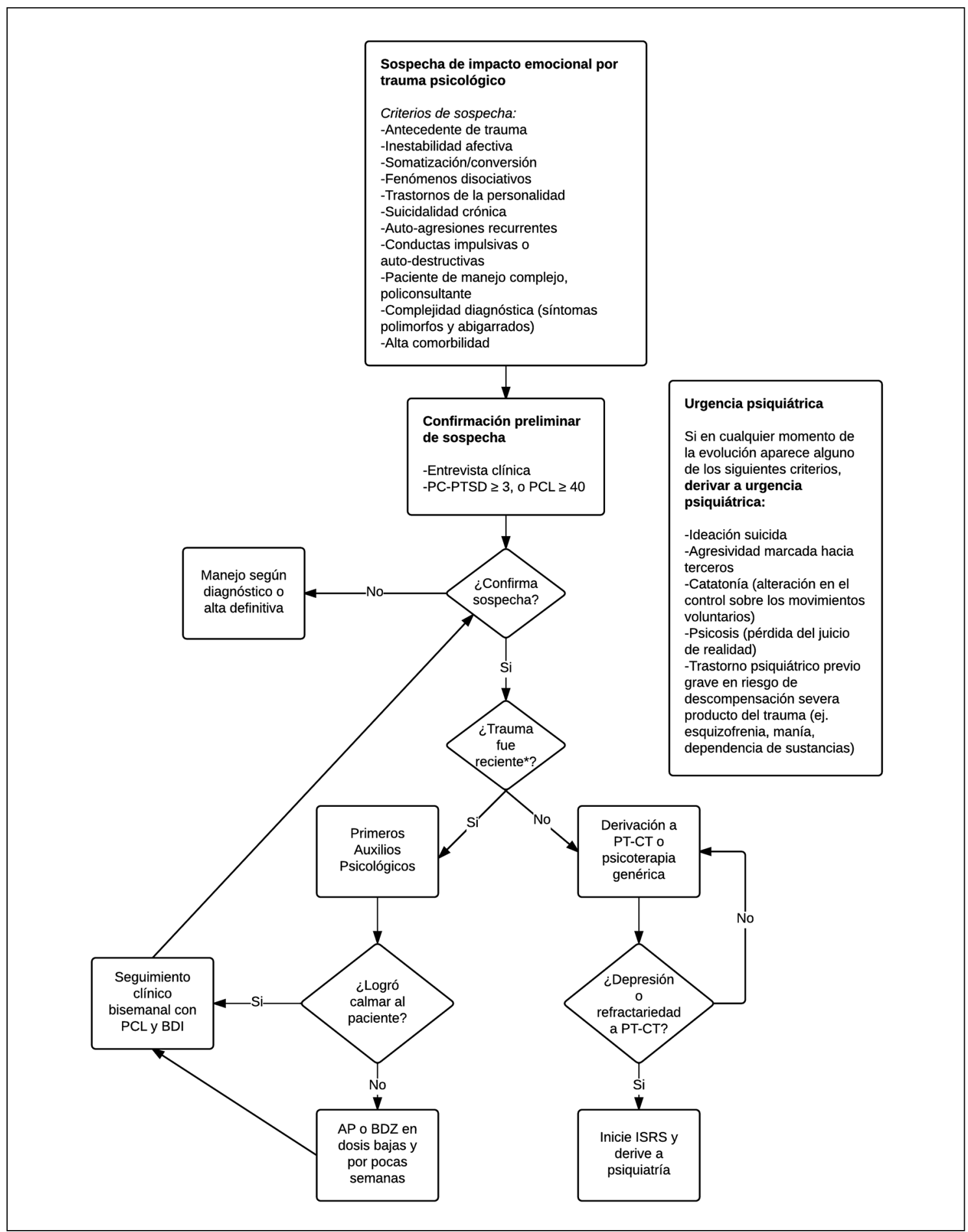

Figura 2. Algoritmo de manejo del trauma psicológico en la atención primaria de salud. AP: antipsicóticos; BDI: Beck Depression Inventory; BDZ: benzodiazepinas; ISRS: inhibidores selectivos de la serotonina; PC-PTSD: primary care PTSD screening; PLC: post-traumatic stress disorder checklist; PT-CT: psicoterapias centradas en el trauma. *Trauma reciente: hace menos de dos semanas. 
Aunque estos instrumentos están diseñados para medir síntomas de trastornos ya consolidados, su aplicación precoz servirá para tener un nivel basal de sintomatología contra la cual objetivar cambio sintomático posterior.

\section{Manejo a partir de la tercera semana post-trauma}

A partir de la tercera semana el manejo dependerá del nivel de disfunción que produzcan los síntomas post-traumáticos y su evolución. Si los síntomas no son muy disruptivos y van disminuyendo paulatinamente (lo que se puede objetivar con las escalas PCL y BDI), bastará con adoptar una conducta expectante, efectuar psicoeducación y hacer seguimiento. Por el contrario, si los síntomas son muy disruptivos, llegando a configurar un Trastorno de Estrés Agudo, un Trastorno de Estrés Postraumático u otro trastorno del espectro postraumático (aunque sólo sea subclínico), o si los síntomas se mantienen estables en el tiempo en vez de disminuir como se esperaría, el tratamiento de elección es alguna de las Psicoterapias Centradas en el Trauma (PT-CT) basadas en la evidencia, como Terapia de Desensibilización y Reprocesamiento por Movimientos Oculares (EMDR, de sus siglas en inglés) o Terapia Cognitivo Conductual Centrada en el Trauma ${ }^{48,49}$. Esta recomendación se fundamenta en que estas herramientas terapéuticas son las que han demostrado mayor efectividad (incluyendo como comparación otras modalidades de psicoterapia y psicofármacos), han sido probadas en diversos escenarios (accidentes vehiculares, desastres naturales, agresiones físicas y sexuales, entre otros), y presentan menores tasas de efectos adversos ${ }^{49}$. Las PT-CT suelen requerir 8 a 15 sesiones semanales de $60 \mathrm{~min}$, por lo que los tratamientos suelen tardar dos a tres meses, logrando tasas de efectividad con números necesarios a tratar $(\mathrm{NNT}) \leq 4$ para casos simples ${ }^{50}$. En las sesiones se le pide al paciente que recuerde con todo detalle el trauma para así desensibilizar y reprocesar las reacciones emocionales en un ambiente seguro y controlado como es el de la consulta. También se incluyen ejercicios de exposición in vivo, que consisten en un enfrentamiento real a situaciones, personas o lugares que puedan estar siendo evitados (ej. pasar por la esquina donde fue el accidente o asalto), lo que se realiza de manera gradual. Además se identifican, cuestionan y modifican los pensamientos, significados e interpretaciones distorsionadas relacionadas con el evento, intentando reemplazarlas por pensamientos más positivos (ej. “yo fui culpable" se reemplaza por "no tenía como saberlo"), se hace psicoeducación y se entregan herramientas de auto-relajación ${ }^{39,51}$. En el caso de EMDR, todo lo anterior es facilitado a través de estimulación bilateral alternante visual, auditiva o táctil ${ }^{52}$, que aunque no se sabe bien como funciona, parece contribuir al reprocesamiento emocional de los recuerdos traumáticos ${ }^{53}$. Lamentablemente en Chile son pocos los profesionales capacitados para aplicar estas intervenciones ${ }^{54}$, por lo que, en su defecto, la alternativa en nuestro medio será la psicoterapia convencional que se aplica habitualmente en consultorios, la que también han demostrado efectividad, aunque menor ${ }^{48}$.

Aquellos pacientes con comorbilidad depresiva y aquellos refractarios a las PT-CT requerirán psicofármacos. En estos casos los Inhibidores Selectivos de la Serotonina (ISRS) son el tratamiento de elección, de preferencia sertralina y paroxetina por contar con mayor evidencia de efectividad ${ }^{49}$. Otros pacientes podrían requerir manejo coadyuvante con antipsicóticos y/o estabilizadores del ánimo (ej. pacientes psicóticos, con trastornos disociativos, con mucha inestabilidad afectiva o muy impulsivos). En todos los casos en que sea necesario adicionar fármacos a la psicoterapia sugerimos derivar a psiquiatría para manejo por especialidad, ya que se podrían requerir intervenciones que se escapan al foco del quehacer de la APS.

\section{Trastorno de estrés postraumático}

El TEPT, aunque no el único, es el cuadro más característico de los trastornos post-trauma. En la Tabla 5 se resumen los síntomas del TEPT de acuerdo al DSM-5 y el CIE-11, este último a ser publicado en 2017. Durante el primer mes post-trauma estos síntomas suelen estar presentes en forma moderada, llegando a considerarse patológicos sólo si son muy intensos y se acompañan de síntomas disociativos como amnesia, despersonalización o desrealización, en cuyo caso se configura un Trastorno de Estrés Agudo. Sólo si los síntomas se mantienen más de un mes se configura un TEPT propiamente tal, lo que resalta el hecho 
Tabla 5. Síntomas del TEPT, de acuerdo al DSM-5 y CIE-11

\begin{abstract}
DSM-5
Presencia de $\geq 1$ síntoma de intrusión recurrente, involuntario y perturbador relacionado al trauma como pesadillas, recuerdos, reacciones disociativas (ej. flashbacks), malestar psicológico y fisiológico frente a claves evocativas del trauma
\end{abstract}

Evitación persistente de $\geq 1$ estímulo relacionado al trauma, como recuerdos, sentimientos o claves externas (ej. personas, lugares, cosas)

Alteraciones negativas en $\geq 2$ cogniciones o emociones, como incapacidad para recordar partes importantes de trauma, disminución del interés de participar en actividades, sentimientos de desapego y distanciamiento de otros, creencias persistentemente negativas, culpa injustificada o un estado afectivo negativo

Alteración significativa del estado de activación o reactividad fisiológica, consistente en $\geq 2$ fenómenos como conducta irritable o agresiva, conducta imprudente o auto-destructiva, hipervigilancia, sobresaltos, desconcentración o alteraciones del sueño

Los síntomas deben durar $\geq 1$ mes

Los síntomas deben causar malestar significativo o deterioro del funcionamiento

Se especifica subtipo disociativo, si existe despersonalización o desrealización significativas

Se especifica subtipo retardado, si comienza después de seis meses post-trauma

*El CIE-11 se espera que sea publicado el año 2017. La versión actual que se presenta corresponde a una versión "beta", es decir en construcción y sujeta a posibles modificaciones.

que este cuadro es un trastorno de la recuperación natural del trauma. Este concepto es clave, porque enfatiza la tendencia espontánea a la recuperación emocional y no patologiza el malestar inicial de las personas luego de un evento traumático.

\section{Sospecha de trastorno de estrés postraumático}

Se debe sospechar secuelas psicológicas de eventos traumáticos en toda persona con inestabilidad afectiva, somatización/conversión, fenómenos disociativos, trastornos de la personalidad, suicidalidad crónica, auto-agresiones, conductas impulsivas o auto-destructivas, pacientes de ma-

\section{CIE-11*}

Reexperimentación del traumas en el presente en forma de recuerdos, flashbacks o pesadillas vívidas e intrusivas, acompañadas de fuertes y abrumadoras emociones como miedo u horror y sensaciones físicas intensas

Evitación de pensamientos, recuerdos, actividades, situaciones o personas evocativas del trauma

Percepción persistente de peligro, manifestada en hipervigilancia o sobresaltos frente a ruidos repentinos

Los síntomas deben durar por lo menos varias semanas

Los síntomas deben producir un deterioro significativo del funcionamiento

Especificar TEPT complejo si se cumple además lo siguiente:

a) La exposición a traumas fue repetida o prolongada y con pocas o ninguna posibilidad de escape (ej. tortura, esclavitud, genocidio en campos de concentración, violencia intrafamiliar prolongada, maltrato infantil sexual o físico repetido)

b) Existen dificultades profundas y graves en la regulación emocional

c) El paciente presenta creencias persistentes acerca del sí mismo como insuficiente, fracasado o incapaz, acompañado de sentimientos de vergüenza, culpa e impotencia en relación al trauma

d) Existen dificultades persistentes para mantener relaciones interpersonales e intimar nejo complejo o policonsultantes, complejidad diagnóstica (síntomas polimorfos o abigarrados) o cuando se cuente con antecedentes de trauma en la biografía. El diagnóstico de TEPT no es fácil cuando es crónico ${ }^{14}$, ya que el antecedente del trauma suele perderse en el tiempo, olvidarse o confundirse entre muchos otros hechos traumáticos. Además los pacientes y los profesionales de la salud suelen evitar hablar de experiencias traumáticas por el malestar que ocasiona hacerlo, o porque simplemente no vinculan esas experiencias con los síntomas actuales, ignorando el hecho que las experiencias traumáticas aumentan el riesgo de enfermedades cardiovasculares, dolor lumbar, asma, problemas estomacales, dolores crónicos 
músculo-esqueléticos, entre otros ${ }^{55,56}$. Una situación que engloba estas características puede ser encontrada en la Tabla 6.

Como muchos de estos pacientes presentan trastornos de la personalidad, en especial del tipo borderline $e^{57}$, pueden producir rechazo por parte de los profesionales de la salud, y resistencia a indagar en su biografía. Esto se ve fuertemente agravado por la escasez de tiempo asignado para cada consulta en la APS. Los pacientes también pueden ocultar sus experiencias traumáticas por vergüenza o culpa, y la alta comorbilidad con trastornos del ánimo, ansiosos, somatomorfos, entre otros, suele complejizar aún más el diagnóstico. Un $80 \%$ de los pacientes con TEPT tienen dos o más diagnósticos asociados, como depresión, abuso de sustancias o fobias ${ }^{8}$. Dado que el perfil del paciente descrito es común en la APS, para facilitar el diagnóstico se recomienda utilizar de forma rutinaria herramientas de tamizaje breve, como la Primary Care PTSD Screen (PC-PTSD) ${ }^{58}$ o la PCL, que han demostrado altos porcentajes de sensibilidad y especificidad ${ }^{59} \mathrm{y}$ en el caso de esta última, se encuentra validada en Chile ${ }^{46}$ (Tabla 7). En aquellos casos en que el puntaje obtenido por el paciente supere el punto de corte se sugiere derivar a psiquiatría para confirmación diagnóstica. Es importante recordar que estos instrumentos sólo son útiles para realizar una detección o tamizaje inicial (screening), y eventualmente, en el caso de la PCL, como herramienta para facilitar el seguimiento sintomático, pero el diagnóstico final siempre debe ser confirmado a través de una entrevista clínica formal por un clínico experimentado.

\section{Consideraciones en niños y adolescentes}

Los niños tienden a recuperarse en forma espontánea luego de un trauma al igual que los adultos, y si desarrollan un TEPT las alternativas de tratamiento son las mismas, aunque poniendo especial énfasis a incorporar a los padres en el tratamiento y ajustar el trabajo al nivel de desarrollo del paciente y su familia ${ }^{60-62}$. Es importante considerar en este grupo etario algunas manifestaciones especiales del TEPT que no suelen observarse en adultos: conductas regresivas como pérdida de control de esfínteres y del lenguaje, rechazo a separarse del cuidador y juegos estereotipados relacionados con el trauma ${ }^{63}$.

\section{Tabla 6. Viñeta clínica}

La señora M. es una mujer de 47 años, separada, con dos hijos de 13 y 17 años. Trabaja como modista en su casa. Tuvo un episodio depresivo autolimitado en su juventud. Acude al consultorio derivada desde la urgencia para control luego de haber sido evaluada allí por dolor precordial intermitente, sin hallazgos positivos al electrocardiograma ni en la evaluación de enzimas cardíacas. Durante la entrevista con su médico, la Sra. M. relata que hace dos meses presenta un dolor opresivo retroesternal especialmente en las noches o cuando está sola, acompañado de cefalea, náuseas y temblor. Su sueño ha estado más interrumpido y hay días en los que siente permanentemente un nudo en la garganta. Indagando con mayor profundidad en sus antecedentes, aparece tangencialmente en la historia un hecho donde la paciente sufrió un grave accidente de tránsito hace cuatro meses, en el que falleció el ocupante del otro vehículo involucrado y ella resultó policontusa. Al contar su experiencia la Sra. M se muestra visiblemente afectada, comienza a temblar y se le humedecen los ojos. Ante el relato, el clínico evita seguir hablando del asunto y sigue adelante con el examen físico

\section{Conclusiones}

Un alto porcentaje de los pacientes que consultan en APS han sido afectados por experiencias traumáticas, y un grupo significativo presenta secuelas emocionales. Lamentablemente estas no suelen diagnosticarse y menos aún tratarse, producto de la variedad de síntomas que conllevan y la escasez de tiempo y disposición de clínicos y pacientes para hablar del tema.

La mayoría de las personas reacciona con un importante malestar durante los primeros días a semanas después de un trauma, el que habitualmente remite al cabo de algunas semanas. En una minoría, sin embargo, los síntomas pueden mantenerse en el tiempo y cronificarse, con altos costos para el individuo, su familia y la sociedad. Lamentablemente aún no sabemos cómo discriminar qué pacientes serán los que queden con secuelas, por lo que en las primeras dos semanas post-trauma sólo deberían brindarse intervenciones inespecíficas como los Primeros Auxilios Psicológicos. Deben evitarse las benzodiazepinas porque en el largo plazo podrían aumentar la incidencia de TEPT.

Deben sospecharse secuelas emocionales de experiencias traumáticas en toda persona con inestabilidad anímica, refractariedad a tratamientos convencionales, somatización, autoagresiones, 


\section{Tabla 7. Recursos útiles sobre trauma psicológico disponibles en internet}

\begin{tabular}{|c|c|}
\hline \multirow[t]{16}{*}{ Para profesionales } & $\begin{array}{l}\text { PTSD Checklist (PCL): Características y descarga del instrumento, National Center for PTSD (EE.UU., } \\
\text { en inglés), disponible en http://goo.gl/Kg81 aZ }\end{array}$ \\
\hline & $\begin{array}{l}\text { Primary Care PTSD Screen (PC-PTSD): Características y descarga del instrumento, National Center } \\
\text { for PTSD (EE.UU., en inglés), disponible en http://goo.gl/BTBrr9 }\end{array}$ \\
\hline & $\begin{array}{l}\text { Sociedad Internacional para el Estudio del Estrés Traumático (portal web, en inglés), disponible en } \\
\text { https://www.istss.org/ }\end{array}$ \\
\hline & $\begin{array}{l}\text { Sociedad Europea para el Estudio del Estrés Traumático (portal web, en inglés), disponible en } \\
\text { https://www.estss.org/ }\end{array}$ \\
\hline & $\begin{array}{l}\text { Sociedad Internacional para el Estudio del Trauma y la Disociación (portal web, en inglés), dispo- } \\
\text { nible en http://www.isst-d.org/ }\end{array}$ \\
\hline & $\begin{array}{l}\text { Centro Nacional de Trastorno de Estrés Postraumático (EE.UU.) (portal web, en inglés), disponible } \\
\text { en http://www.ptsd.va.gov/ }\end{array}$ \\
\hline & $\begin{array}{l}\text { Eliminar la violencia contra los niños y niñas: Seis estrategias para la acción, de UNICEF, disponible } \\
\text { en http://goo.gl/Ngxwam }\end{array}$ \\
\hline & $\begin{array}{l}\text { Abuso sexual infantil: Cuestiones relevantes para su tratamiento en la justicia, de UNICEF, disponible } \\
\text { en http://goo.gl/mkXnkL }\end{array}$ \\
\hline & $\begin{array}{l}\text { Preguntas más frecuentes sobre trauma y disociación para estudiantes y profesionales en forma- } \\
\text { ción, de International Society for the Study of Trauma and Dissociation, disponible en http://goo. } \\
\text { gl/QIHbzl }\end{array}$ \\
\hline & $\begin{array}{l}\text { Revista Iberoamericana de Psicotraumatología y Disociación, disponible en http://revibapst.com/ } \\
\text { home/ }\end{array}$ \\
\hline & $\begin{array}{l}\text { Recursos gratis (en inglés): Conjunto de vídeos y presentaciones sobre distintos tópicos de estrés } \\
\text { traumático, de International Society for Traumatic Stress Studies, disponible en http://goo.gl/18fLKT }\end{array}$ \\
\hline & $\begin{array}{l}\text { Primeros Auxilios Psicológicos: Guía de operaciones prácticas, } 2^{a} \text { edición, de National Child Trauma- } \\
\text { tic Stress Network y National Center for PTSD (EE.UU.), disponible en http://goo.gl/CUJJqq }\end{array}$ \\
\hline & $\begin{array}{l}\text { Primera ayuda psicológica: Guía para trabajadores de campo, de la Organización Mundial de la } \\
\text { Salud, War Trauma Foundation y Visión Mundial Internacional, disponible en http://goo.gl/My3iUG }\end{array}$ \\
\hline & $\begin{array}{l}\text { Guías clínicas para el manejo de condiciones específicamente relacionadas a estrés (en inglés), de } \\
\text { la Organización Mundial de la Salud, disponible en http://goo.gl/W1Vw2D }\end{array}$ \\
\hline & $\begin{array}{l}\text { Guías clínicas sobre Trastorno de Estrés Postraumático en adultos y niños en el nivel primario y } \\
\text { secundario de salud (en inglés), The National Institute for Clinical Excellence (NICE - Reino Unido), } \\
\text { disponible en http://goo.gl/7H1XXL }\end{array}$ \\
\hline & $\begin{array}{l}\text { Introducción al trauma psíquico, sus secuelas y su tratamiento, Dr. Rodrigo Figueroa, Unidad de } \\
\text { Trauma y Disociación, Escuela de Medicina UC, disponible en https://goo.gl/Tm4yaV }\end{array}$ \\
\hline \multirow[t]{3}{*}{$\begin{array}{l}\text { Para padres y } \\
\text { cuidadores }\end{array}$} & $\begin{array}{l}\text { Trinka y Juan - El día que la tierra se movió. Cuento para niños y guía para padres de familia } \\
\text { afectadas por terremotos, de National Child Traumatic Stress Network, disponible en http://goo. } \\
\text { gl/536d2g }\end{array}$ \\
\hline & $\begin{array}{l}\text { Los niños y el trauma, de International Society for Traumatic Stress Studies, disponible en http:// } \\
\text { goo.gl/Segda1 }\end{array}$ \\
\hline & $\begin{array}{l}\text { Disociación en niños y adolescentes: Preguntas más comunes formuladas por los padres, de In- } \\
\text { ternational Society for the Study of Trauma and Dissociation, disponible en http://goo.gl/HgzGYl }\end{array}$ \\
\hline \multirow[t]{5}{*}{$\begin{array}{l}\text { Para adultos } \\
\text { afectados }\end{array}$} & $\begin{array}{l}\text { Cuando un amigo o alguien a quien se quiere ha sido traumatizado, de International Society for } \\
\text { Traumatic Stress Studies, disponible en http://goo.gl/GQAuHL }\end{array}$ \\
\hline & $\begin{array}{l}\text { Desastres masivos, trauma y pérdida, de International Society for Traumatic Stress Studies, dispo- } \\
\text { nible en http://goo.gl/NHglzL }\end{array}$ \\
\hline & $\begin{array}{l}\text { Relación entre los síntomas del trauma y los problemas del uso de sustancias, de International } \\
\text { Society for Traumatic Stress Studies, disponible en http://goo.gl/4A5JJU }\end{array}$ \\
\hline & $\begin{array}{l}\text { Violencia en relaciones íntimas, de International Society for Traumatic Stress Studies, disponible } \\
\text { en http://goo.gl/R39e5G }\end{array}$ \\
\hline & $\begin{array}{l}\text { Recuerdo del trauma sufrido durante la infancia, de International Society for Traumatic Stress } \\
\text { Studies, disponible en http://goo.gl/v09zZ5 }\end{array}$ \\
\hline
\end{tabular}


suicidalidad crónica, trastornos de la personalidad y síntomas vagos y abigarrados, así como en aquellos en los que se conozca del antecedente traumático. La confirmación se puede facilitar con instrumentos breves de tamizaje como el PC-PTSD o el PCL.

A partir de la tercera semana aquellos pacientes que persistan con síntomas que comprometan el funcionamiento deben ser derivados a PT-CT o en su defecto a psicoterapia convencional. Si además presentan depresión o si no responden a la psicoterapia se les debe indicar un ISRS y derivar a psiquiatría.

\section{Referencias}

1. American Psychological Association (APA). Diagnostic and Statistical Manual of Mental Disorders (DSM- $5^{\circledR}$ ). 2013.

2. World Health Organization (WHO). Post-traumatic stress disorder [Internet]. ICD-11 Beta Draft (Joint Linearization for Mortality and Morbidity Statistics). 2015 [consultado el 2015 Dec 28]. Disponible en: http:// id.who.int/icd/entity/2070699808

3. Stein DJ, McLaughlin KA, Koenen KC, Atwoli L, Friedman MJ, Hill ED, et al. DSM-5 and ICD-11 definitions of posttraumatic stress disorder: investigating "narrow" and "broad" approaches. Depress Anxiety 2014; 31 (6): 494-505.

4. Zlotnick C, Johnson J, Kohn R, Vicente B, Rioseco P, Saldivia S. Epidemiology of trauma, post-traumatic stress disorder (PTSD) and co-morbid disorders in Chile. Psychol Med 2006; 36 (11): 1523-33.

5. Norris FH, Murphy AD, Baker CK, Perilla JL, Rodríguez FG, Rodríguez J de J. Epidemiology of trauma and posttraumatic stress disorder in Mexico. J Abnorm Psychol 2003; 112 (4): 646-56.

6. Perkonigg A, Kessler RC, Storz S, Wittchen HU. Traumatic events and post-traumatic stress disorder in the community: prevalence, risk factors and comorbidity. Acta Psychiatr Scand 2000; 101 (1): 46-59.

7. Creamer M, Burgess P, McFarlane AC. Post-traumatic stress disorder: findings from the Australian National Survey of Mental Health and Well-being. Psychol Med 2001; 31 (7): 1237-47.

8. Kessler RC, Sonnega A, Bromet E, Hughes M, Nelson CB. Posttraumatic stress disorder in the National Comorbidity Survey. Arch Gen Psychiatry 1995; 52 (12): 1048-60.

9. Moreau C, Zisook S. Rationale for a posttraumatic stress spectrum disorder. Psychiatr Clin North Am 2002; 25 (4): 775-90.

10. Kessler RC. Posttraumatic stress disorder: the burden to the individual and to society. J Clin Psychiatry 2000; 61 Suppl 5: 4 .

11. Waddington A, Ampelas J-FF, Mauriac F, Bronchard M, Zeltner L, Mallat V. Post-traumatic stress disorder (PTSD): the syndrome with multiple faces. Encephale 2003; 29 (1): 20-7.

12. Galatzer-Levy IR, Bryant RA. 636,120 Ways to Have Posttraumatic Stress Disorder. Perspect Psychol Sci 2013; 8 (6): 651-62.

13. Wade D, Howard A, Fletcher S, Cooper J, Forbes D. Early response to psychological trauma-what GPs can do. Aust Fam Physician 2013; 42 (9): 610-4.

14. Grinage BD. Diagnosis and management of post-traumatic stress disorder. Am Fam Physician 2003; 68 (12): 2401-8.

15. Stein MB, McQuaid JR, Pedrelli P, Lenox R, Mccahill ME. Posttraumatic stress disorder in the primary care medical setting. Gen Hosp Psychiatry 2000; 22 (4): 2619.

16. Ministerio de Salud de Chile. Estudio de carga de enfermedad y carga atribuible [Internet]. Ministerio de Salud de Chile. 2008 [consultado el 28 de marzo de 2016]. Disponible en: http://epi.minsal.cl/wp-content/ uploads/2016/04/Informe-final-carga_Enf_2007.pdf

17. Mathers C, Fat D, Boerma J. The global burden of disease: 2004 update. 2008 [consultado el 28 de diciembre de 2015]; Disponible en: http://www.who.int/healthinfo/ global_burden_disease/GBD_report_2004update_full. pdf

18. Monson CM, Taft CT, Fredman SJ. Military-related PTSD and intimate relationships: from description to theory-driven research and intervention development. Clin Psychol Rev 2009; 29 (8): 707-14.

19. Taft CT, Watkins LE, Stafford J, Street AE, Monson CM. Posttraumatic stress disorder and intimate relationship problems: a meta-analysis. J Consult Clin Psychol 2011; 79 (1): 22-33.

20. McFarlane AC. Military deployment: the impact on children and family adjustment and the need for care. Curr Opin Psychiatry 2009; 22 (4): 369-73.

21. Sayers SL, Farrow VA, Ross J, Oslin DW. Family problems among recently returned military veterans referred for a mental health evaluation. J Clin Psychiatry 2009; 70 (2): 163-70.

22. Wilcox HC, Storr CL, Breslau N. Posttraumatic stress disorder and suicide attempts in a community sample of urban american young adults. Arch Gen Psychiatry 2009; 66 (3): 305-11. 
23. Breslau N. The epidemiology of posttraumatic stress disorder: what is the extent of the problem? J Clin Psychiatry 2001; 62 Suppl 1: 16-22.

24. Ozer EJ, Best SR, Lipsey TL, Weiss DS. Predictors of posttraumatic stress disorder and symptoms in adults: a meta-analysis. Psychol Bull 2003; 129 (1): 52-73.

25. Brewin CR, Andrews B, Valentine JD. Meta-analysis of risk factors for posttraumatic stress disorder in trauma-exposed adults. J Consult Clin Psychol 2000; 68 (5): 748-66.

26. Bryant R, Creamer M, O’Donnell M, Silove D, McFarlane A. The capacity of acute stress disorder to predict posttraumatic psychiatric disorders. J Psychiatr Res 2012; 46 (2): 168-73.

27. Bryant RA, Creamer M, O’Donnell M, Silove D, Mcfarlane AC, Forbes D. A Comparison of the Capacity of DSM-IV and DSM-5 Acute Stress Disorder Definitions to Predict Posttraumatic Stress Disorder and Related Disorders. J Clin Psychiatry 2015; 391-7.

28. Green BL, Lindy JD, Grace MC, Leonard AC. Chronic posttraumatic stress disorder and diagnostic comorbidity in a disaster sample. J Nerv Ment Dis; 1992; 180 (12): 760-6.

29. Figueroa RA, Marín H, González M. Psychological support for disaster victims: an evidence-based care model. Rev Med Chile 2010; 138 (2): 143-51.

30. Figueroa RA, Cortés PF. Propuesta para abordar los problemas de salud mental detectados en el proceso diagnóstico de la Delegación Presidencial para la reconstrucción tras el terremoto del 27 de febrero de 2010. Rev Med Chile 2016; 144: 247-52.

31. Effects of Traumatic Stress after Mass Violence, Terror, or Disaster-PTSD: National Center for PTSD. 2015 Aug 17 [consultado el 2015 Dec 28]; Disponible en: http:// www.ptsd.va.gov/professional/trauma/disaster-terrorism/stress-mv-t-dhtml.asp

32. Bonanno GA. Loss, trauma, and human resilience: have we underestimated the human capacity to thrive after extremely aversive events? Am Psychol 2004; 59 (1): 20-8.

33. Tedeschi RG, Calhoun LG. The Posttraumatic Growth Inventory: measuring the positive legacy of trauma. J Trauma Stress 1996; 9 (3): 455-71.

34. Agorastos A, Marmar CR, Otte C. Immediate and early behavioral interventions for the prevention of acute and posttraumatic stress disorder. Curr Opin Psychiatry 2011; 24 (6): 526-32.

35. Organización Mundial de la Salud, War Trauma Foundation, World Vision Internacional. Primera ayuda psicológica: Guía para trabajadores de campo. Ginebra: OMS; 2012.
36. Figueroa RA, Repetto P, Bernales M. Incorporation of psychological first aid to the undergraduate curriculum. Rev Med Chile 2014; 142 (7): 934-5.

37. Hobfoll SE, Watson P, Bell CC, Bryant RA, Brymer MJ, Friedman MJ, et al. Five essential elements of immediate and mid-term mass trauma intervention: empirical evidence. Psychiatry 2007; 70 (4): 269-83.

38. Robertson K. Active listening: more than just paying attention. Aust Fam Physician 2005; 34 (12): 1053-5.

39. Foa E, Hembree E, Rothbaum BO. Prolonged exposure therapy for PTSD: Emotional processing of traumatic experiences therapist guide. Oxford University Press; 2007.

40. Reger GM, Hoffman J, Riggs D, Rothbaum BO, Ruzek J, Holloway KM, et al. The "PE coach" smartphone application: An innovative approach to improving implementation, fidelity, and homework adherence during prolonged exposure. Psychol Serv 2013; 10 (3): 342-9.

41. Jacobson R. Pocket Therapy. Sci Am Mind. Scientific American, Inc. 2014; 25 (6): 72-3.

42. Progressive Relaxation: A Physiological and Clinical Investigation of Muscular States and Their Significance in Psychology and Medical Practice. J Am Med Assoc. American Medical Association 1938; 111 (12): 1129.

43. Blanaru M, Bloch B, Vadas L, Arnon Z, Ziv N, Kremer $\mathrm{I}$, et al. The effects of music relaxation and muscle relaxation techniques on sleep quality and emotional measures among individuals with posttraumatic stress disorder. Ment Illn 2012; 4 (2): e13.

44. Guina J, Rossetter S. Benzodiazepines for PTSD: a systematic review and meta-analysis. J Psychiatr 2015; 21 (4): 281-303.

45. Barbui C, Gastaldon C, Cipriani A. Benzodiazepines and risk of dementia: true association or reverse causation? Epidemiol Psychiatr Sci 2013; 22 (4): 307-8.

46. Vera-Villarroel P, Zych I, Celis-Atenas K, Córdova-Rubio N, Buela-Casal G. Chilean validation of the Posttraumatic Stress Disorder Checklist-Civilian version (PCL-C) after the earthquake on February 27, 2010. Psychol Rep 2011; 109 (1): 47-58.

47. Sanz J, Vázquez C. Fiabilidad, validez y datos normativos del inventario para la depresión de Beck. Psicothema 1998; 10 (2): 303-18.

48. Bisson JI, Roberts NP, Andrew M, Cooper R, Lewis C. Psychological therapies for chronic post-traumatic stress disorder (PTSD) in adults. Cochrane database Syst Rev 2013; 12: CD003388.

49. Difede J, Olden M, Cukor J. Evidence-based treatment of post-traumatic stress disorder. Annu Rev Med Annual Reviews 2014; 65: 319-32.

50. Gartlehner G, Forneris CA, Brownley KA, Gaynes BN, 
Sonis J, Coker-Schwimmer E, et al. Interventions for the Prevention of Posttraumatic Stress Disorder (PTSD) in Adults After Exposure to Psychological Trauma. Rockville (MD): Agency for Healthcare Research and Quality (US); 2013.

51. Cognitive Processing Therapy for Post-Traumatic Stress Disorder. Society of Clinical Psychology [Internet]. [consultado el 2015 Dec 28]. Disponible en: https:// www.div12.org/psychological-treatments/disorders/ post-traumatic-stress-disorder/cognitive-processing-therapy/

52. Shapiro F. Eye movement desensitization and reprocessing: Basic principles, protocols, and procedures. The Guilford Press; 2001.

53. Lee CW, Cuijpers P. A meta-analysis of the contribution of eye movements in processing emotional memories. J Behav Ther Exp Psychiatry 2013; 44 (2): 231-9.

54. Terapeutas. EMDR Chile [Internet] [consultado el 28 de diciembre de 2015]. Disponible en: http://www. emdrchile.cl/terapeutas/

55. McFarlane AC. The long-term costs of traumatic stress: intertwined physical and psychological consequences. World Psychiatry 2010; 9 (1): 3-10.

56. Glaesmer H, Brähler E, Gündel H, Riedel-Heller SG. The association of traumatic experiences and posttraumatic stress disorder with physical morbidity in old age: a German population-based study. Psychosom Med 2011; 73 (5): 401-6.

57. Harned MS, Rizvi SL, Linehan MM. Impact of co-occurring posttraumatic stress disorder on suicidal women with borderline personality disorder. Am J Psychiatry 2010; 167 (10): 1210-7.
58. Prins A, Ouimette P, Kimerling R, Camerond RP, Hugelshofer DS, Shaw-Hegwer J, et al. The primary care PTSD screen (PC-PTSD): development and operating characteristics. Prim Care Psychiatry 2004; 9 (1): 9-14.

59. Spoont MR, Williams JW, Kehle-Forbes S, Nieuwsma JA, Mann-Wrobel MC, Gross R. Does This Patient Have Posttraumatic Stress Disorder?: Rational Clinical Examination Systematic Review. JAMA 2015; 314 (5): 501-10.

60. Silverman WK, Ortiz CD, Viswesvaran C, Burns BJ, Kolko DJ, Putnam FW, et al. Evidence-based psychosocial treatments for children and adolescents exposed to traumatic events. J Clin Child Adolesc Psychol 2008; 37 (1): 156-83.

61. Taylor TL, Chemtob CM. Efficacy of treatment for child and adolescent traumatic stress. Arch Pediatr Adolesc Med 2004; 158 (8): 786-91.

62. Gillies D, Taylor F, Gray C, O’Brien L, D’Abrew N. Psychological therapies for the treatment of post-traumatic stress disorder in children and adolescents. Cochrane Database Syst Rev 2012; 12: CD006726.

63. PTSD in Children and Teens-PTSD: National Center for PTSD. 2015 Aug 13 [consultado el 28 de diciembre de 2015]; Disponible en: http://www.ptsd.va.gov/public/ family/ptsd-children-adolescents.asp

64. Spiegel D, Loewenstein RJ, Lewis-Fernández R, Sar V, Simeon D, Vermetten E, et al. Dissociative disorders in DSM-5. Depress Anxiety 2011; 28 (12): E17-45.

65. Common Reactions After Trauma-PTSD: National Center for PTSD. 2015 Aug 13 [consultado el 28 de diciembre de 2015]; Disponible en: http://www.ptsd.va.gov/ public/problems/common-reactions-after-trauma.asp 